\title{
Security Detection of Building Structure Based on Sparse Encoding Deep Learning Algorithm
}

\author{
Zheng $\mathrm{Li}^{1}$ \\ ${ }^{1}$ Department of Information Engineering, Sichuan College of Architectural \\ Technology, Deyang 618000, China \\ 121428073@qq.com
}

\begin{abstract}
Most health problems of building structures are accumulative damages which are difficult to detect, and it is more difficult to monitor the structure health due to the complexity of the practical structure and the environment noise, and the existing methods need lots of data for model training but it is very complicated to mark the data in practice. In order to solve above problems, the wireless sensor network is configured and the sparse encoding method is adopted to monitor the bridge structure health, and meanwhile the sparse encoding algorithm is adopted for training on the basis of the characteristic extraction of many unlabeled instances, thus to compress data dimensionality and preprocess unlabeled data. Then, the deep learning algorithm is adopted to predict the bridge structure health monitoring type, and meanwhile Hessian optimization is improved on the basis of the linear conjugate gradient in order to replace uncertain Hessian matrix by positive semidefinite Gaussian - Newton curvature matrix for secondary objective combination, thus to improve the efficiency of the deep learning algorithm. The experiment result shows that the security detection of the bridge structure based on deep learning algorithm can monitor the high-accuracy structure health conditions under the sparse encoding of the environment noise.
\end{abstract}

Keywords: Structure security; Deep learning; Sparse encoding; Wireless sensor; Bridge structure

\section{Introduction}

Currently, the structural security detection problem of buildings is a serious problem. For example, bridges may suffer from structural violation due to such factors as continuous traffic, wind load, material aging, environment corrosion and earthquake, thus causing structure defects and damages and significantly shortening the service life of the structure[1 2]. Sometimes, these damages which are different to discover may cause serious security accidents such as collapse and subsidence, or cause serious casualty and property loss. For example, the collapse of Harbin Yang Ming Tan Bridge in August 2012 crashed several vehicles and caused three persons to die; Hunan Tuojiang Bridge under construction suddenly collapsed and caused 64 workers to die, etc. Many facts show that it is very necessary to continuously monitor the structural health conditions in order to avoid such accidents. In order to solve this problem, the research on SHM (Structural Health Monitoring) technology has been widely concerned [3 4]. SHM research in early period is mainly concentrated in the simulation of real physical structures, namely the so called model driven method [5]. This method mainly aims at adopting the mathematical modeling 
and the physical laws to represent the monitored structures. In allusion to the security monitoring problem of the bridge structure in this paper, Hessian optimization is improved according to the proposed sparse encoding characteristics and on the basis of the linear conjugate gradient in order to replace uncertain Hessian matrix by positive semidefinite Gaussian - Newton curvature matrix for secondary objective combination, thus to propose SCDS (Sparse Coding Depth Study for Unlabeled Modal Characteristics) algorithm and improve the efficiency of the deep learning algorithm. Moreover, such algorithm has been applied to the bridge structure security monitoring and the algorithm effectiveness has been also verified through implementation.

\section{Sparse encoding deep learning}

\subsection{Sparse encoding learning}

Another module in the second layer is the sparse encoding module. As mentioned above, there are less labeled data, so the method able to make full use of many unlabeled data and automatically capture the characteristics of the input data should be regarded as the standard for the algorithm selection. The sparse encoding algorithm, as an unsupervised learning algorithm, can meet all above requirements, wherein the sparse encoding algorithm is firstly proposed by Olshausen as the solution for the unsupervised low-level sense calculation model. Hereby, it refers to the system architecture for sparse encoding:

The sparse encoding includes three layers: input layer, hidden layer and output layer. Each nerve cell is connected to the sub-layer nerve cell through weight connection. For the given unlabeled data training set $\left(x^{(1)}, x^{(2)}, x^{(3)}, \cdots\right\}, x^{(i)} \in R^{n}$ is true. The sparse encoding method proposed in Literature [6] is based on BP algorithm, and the input is the target set value, thus indicating the learning approximation of this sparse encoding for function $h_{w, b}(x) \approx x$. If relevant constraints are added to the network to limit the quantity of the hidden cells, then this network can be represented as the input compression representation learning, and the characteristic learning can be adopted for the similarity reconstruction of the input data. In practice, the quantity of the hidden cells is not limited; oppositely, the sparse constraints of the hidden cells are strengthened to limit the quantity of the "active" cells. Generally, if the output of a nerve cell is approximate to 1, then this layer can be regarded as an "active layer"; or else, this layer is regarded as an "inactive layer". Actually, we need to limit the inactive nerve cells of the hidden layers in most time, as shown in Figure 1.

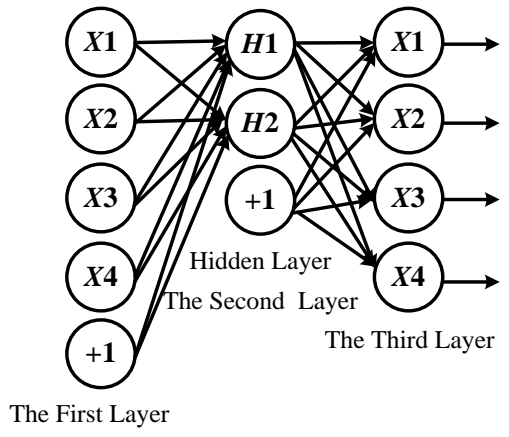

Figure 1. System architecture of sparse encoding 
$a_{j}^{(2)}(x)$ is assumed to represent the activeness of hidden layer $j$ of given input $x$, and the activation function of the hidden layer can be represented as follows during the forward propagation process:

$$
\hat{\rho}_{j}=\frac{1}{m} \sum_{i=1}^{m}\left[a_{j}^{(2)}\left(x^{(i)}\right)\right]
$$

We expect to make $\hat{\rho}_{j}$ approximate to small value $\rho$, namely the so called sparseness parameter. For such implementation, it is necessary to add an additional item to the objective function in order to punish the deviation of $\hat{\rho}_{j}$ to $\rho$. In this paper, KL divergence is selected as the penalty item:

$$
\sum_{j=1}^{L_{2}} \rho \log \frac{\rho}{\hat{\rho}_{j}}+(1-\rho) \log \frac{1-\rho}{1-\hat{\rho}_{j}}
$$

The cost function of BP neural network can be defined as follows:

$$
\begin{aligned}
C(W, b) & =\left[\frac{1}{m} \sum_{i=1}^{m}\left(\frac{1}{2}\left\|h_{w, b}\left(x^{(j)}\right)-y^{(i)}\right\|^{2}\right)\right] \\
& +\frac{\lambda}{2} \sum_{l=1}^{n_{l}-1} \sum_{i=l}^{m_{l}} \sum_{j=1}^{m_{l+1}}\left(w_{i j}^{(t)}\right)^{2}
\end{aligned}
$$

Therefore, the cost function is the sparse encoding and can be modified as follows:

$$
\left\{\begin{array}{l}
C_{\text {sparse }}(W, b)=C(W, b)+\beta \sum_{j=1}^{L_{2}} K L\left(p \| \hat{p}_{j}\right) \\
K L\left(\rho \| \hat{\rho}_{j}\right)=\rho \log \frac{\rho}{\hat{\rho}_{i}}+(1-\rho) \log \frac{1-\rho}{1-\hat{\rho}_{i}}
\end{array}\right.
$$

After the unlabeled data are adopted to establish the sparse encoding for characteristic extraction, relevant classifier can be adopted to predict the bridge structure state. So far, many classification algorithms can be used. In this paper, in consideration of algorithm performance and stability, the deep learning algorithm is selected for characteristic learning and classification. The sparse encoding process mentioned above is as shown in Algorithm 1.

\begin{tabular}{l}
\hline Algorithm 1: BP Sparse Encoding Process \\
\hline Input: training sample $(x, y)$; \\
1. Randomly initialize parameters $W$ and $b$; \\
2. Execute forward propagation calculation: $\hat{\rho}_{j}$; \\
3. for $i \in$ output layer do \\
4. $\delta_{i}^{\left(n_{l}\right)}=\frac{\delta}{\partial z_{i}^{\left(n_{l}\right)}} \frac{1}{2} \square h_{W, b}(x)-y \square^{2}=-\left(y_{i}-a_{i}^{n_{l}}\right) \cdot f(x) ;$ \\
4. $\delta_{i}^{\left(n_{l}\right)}=\frac{\delta}{\partial z_{i}^{\left(n_{l}\right)}} \frac{1}{2} \square h_{W, b}(x)-y \sqsubset^{2}=-\left(y_{i}-a_{i}^{n_{l}}\right) \cdot f(x)$ \\
5. endfor \\
5. endfor \\
6. for $l=n_{l}-1, \cdots, 2$ do \\
6. for $l=n_{l}-1, \cdots, 2$ do \\
7. for $i \in l$ do \\
7. for $i \in l$ do
\end{tabular}


Algorithm 1: BP Sparse Encoding Process

8.

$$
\begin{aligned}
\delta_{i}^{(l)} & =\frac{\delta C}{\partial a_{i}^{(l)}} \frac{\delta a_{i}^{(l)}}{\delta z_{i}^{(l)}} \\
& =\left(\sum_{j=1}^{m_{t+1}} \delta_{j}^{l+1} w_{i j}^{l}+\beta\left(-\frac{\rho}{\hat{\rho}_{j}}+\frac{1-\rho}{1-\hat{\rho}_{j}}\right)\right) \cdot f^{\prime}\left(z_{i}^{(l)}\right) \\
\delta_{i}^{(l)}= & \frac{\delta C}{\partial a_{i}^{(l)}} \frac{\delta a_{i}^{(l)}}{\delta z_{i}^{(l)}} \\
= & \left(\sum_{j=1}^{m_{+1}} \delta_{j}^{l+1} w_{i j}^{l}+\beta\left(-\frac{\rho}{\hat{\rho}_{j}}+\frac{1-\rho}{1-\hat{\rho}_{j}}\right)\right) \cdot f^{\prime}\left(z_{i}^{(l)}\right)
\end{aligned}
$$

8.

9. endfor

9. endfor

10. endfor

10. endfor

11. Calculate the partial derivative

12. Output:

13. $\frac{\delta}{\delta w_{i j}^{(l)}} C(W, b)=\delta_{j}^{(l+1)} \alpha_{l}^{(l)}$;

13. $\frac{\delta}{\delta w_{i j}^{(l)}} C(W, b)=\delta_{j}^{(l+1)} \alpha_{l}^{(l)}$

14. $\frac{\partial}{\delta b_{l}^{(l)}} C(W, b)=\delta_{j}^{(l+1)}$ 。

14. $\frac{\partial}{\delta b_{l}^{(l)}} C(W, b)=\delta_{j}^{(l+1)}$

\subsection{Deep learning algorithm}

\subsubsection{Deep encoding}

Deep encoding, as the learning transformation of DNNs (Deep Neural Networks), is mainly used for dimensionality compression and characteristic extraction. Multiple layers of hidden cells are added to the input and output layers of DNNs for network construction, wherein the node quantity of the central hidden layer should be less than that of the input layer (encoder) and the output layer (decoder). The generality mapping between the input and the output of the model is established through network training. For data dimensionality compression, the common method is PCA (Principal Component Analysis), but the deep encoding method is indicated in Literature [7] to be superior to PCA encoding method.

In order to realize DNNs network training, an unsupervised learning algorithm is firstly proposed in Literature [8] to finely adjust the network parameters through the layer-bylayer greed unsupervised training, thus to conquer the unsatisfactory local optimum value of the deep model. Different from the above literature, the second-order optimization is introduced in this paper to optimize Hessian matrix for deep network training. The structure of the deep neural network is as shown in Figure 2. 


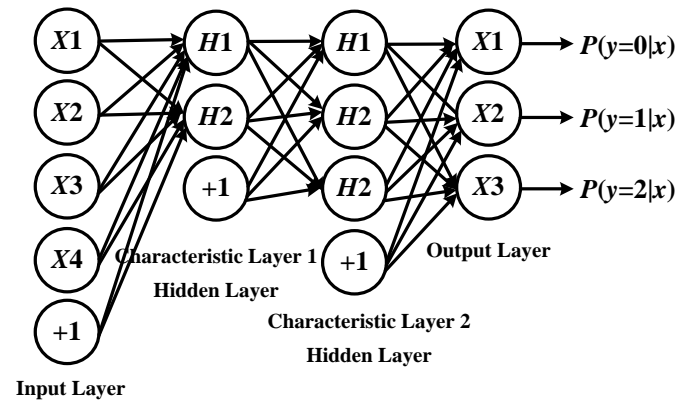

Figure 2. Structure of Deep Neural Network

Slightly different from the system architecture of the sparse encoding in Figure 1, the structure of the deep neural network in Figure 2 is additionally provided with one or more hidden layers for characteristic learning, and such structure is favorable for improving algorithm performance.

\subsubsection{Hessian matrix optimization}

Hessian matrix optimization process mentioned here is sourced from Newton numerical optimization technology. In the typical second-order optimization scheme, such as Newton numerical optimization method, gradient vector $p$ is calculated through iteration and parameter $\theta \in R N$ of objective function $f$ is updated. If the learning parameter is set as $\alpha$, the updating process of parameter $\theta$ can be represented as follows:

$$
\theta_{n+1}=\theta_{n}+\alpha p_{n}
$$

The core of Newton method lies in the local approximation for objective function $f$ around parameter $\theta$, and the implementation process is as follows:

$$
M_{\theta_{n}}(\theta)=f\left(\theta_{n}\right)+\nabla f\left(\theta_{n}\right)^{T} p_{n}+\frac{1}{2} p_{n}^{T} B_{\theta_{n} P_{n}}
$$

Where $B_{\theta_{n}}$ represents damping Hessian matrix of objective function $f$ near $\theta_{n}$; Due to the uncertainty of $\mathrm{H}$, Hessian matrix can be redefined as follows:

$$
B_{\theta_{n}}=H\left(\theta_{n}\right)+\lambda I
$$

Where $\lambda \geq 0$ represents the damping parameter, and $\mathrm{I}$ is the unit matrix. Based on standard Newton method, $M_{\theta_{n}}(\theta)$ is adopted for relevant calculation through the optimization of NXM matrix $B_{\theta_{n}}$ in order to solve system $B_{\theta_{n} P_{n}}=-\nabla f\left(\theta_{n}\right)^{T}$. However, such calculation is very complicated for large $\mathrm{N}$ value, and such complexity has high costs even for medium-scale neural network.

In order to conquer above disadvantages, Hessian optimization is improved on the basis of the linear conjugate gradient in order to replace uncertain Hessian matrix by positive semidefinite Gaussian - Newton curvature matrix for secondary objective combination. The calculation process of this algorithm is as shown in Algorithm 2.

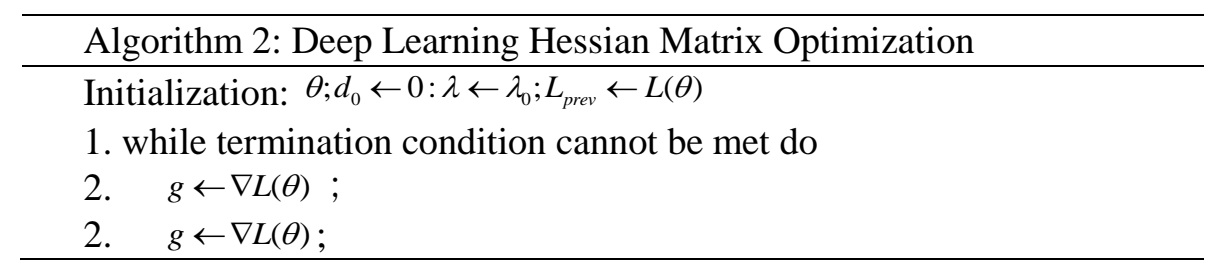




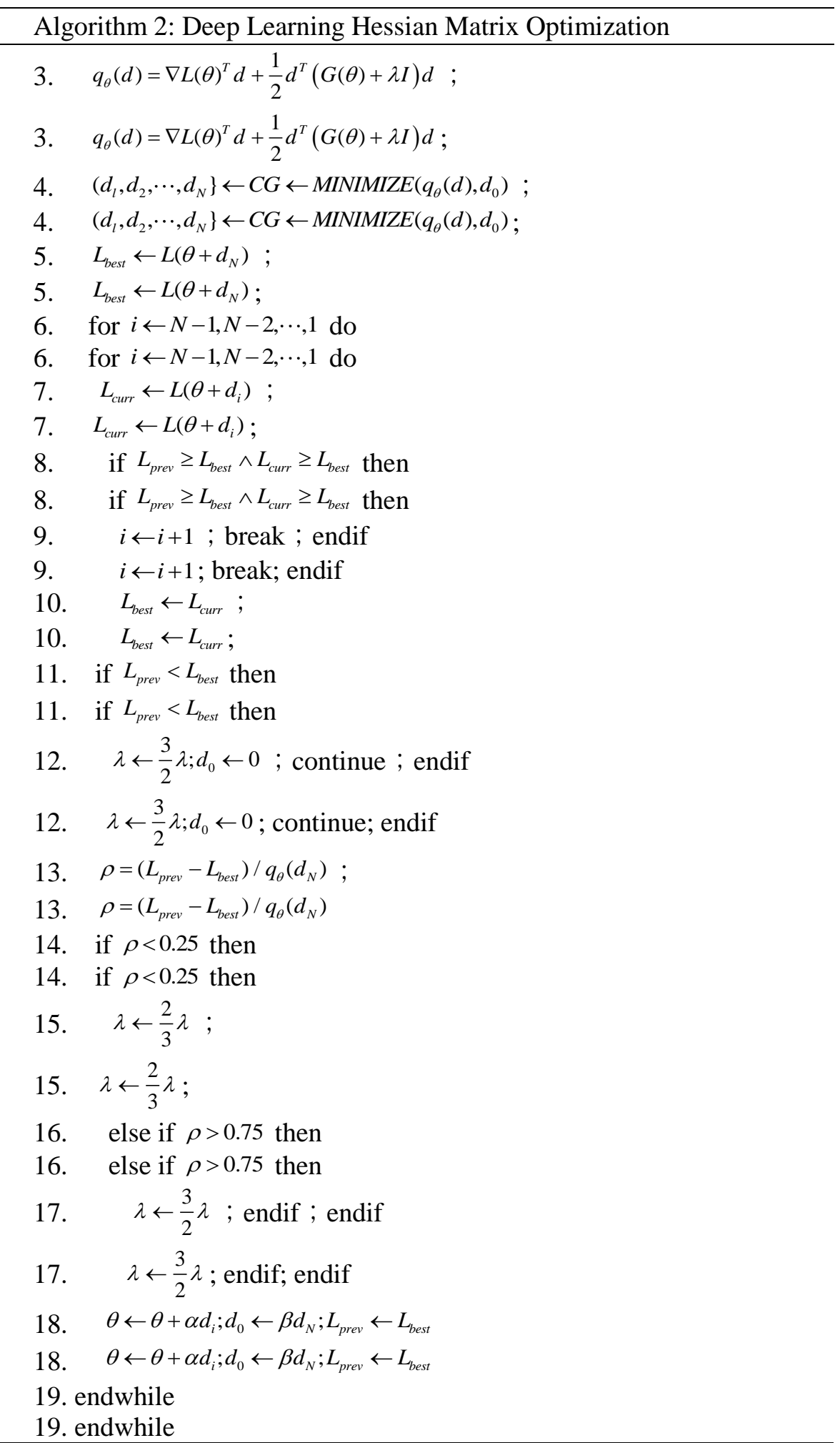

\subsection{Algorithm process description}

The sparse encoding deep learning process is specifically described as follow: 
Step 1: (Sparse characteristic construction) For a group of given training samples, these data are regarded as the sparse encoding inputs, and Algorithm 1 is adopted for characteristic extraction:

$$
f=\left\{f_{l}^{(1)}, f_{l}^{(2)}, \cdots, f_{l}^{(m)}\right\}
$$

Step 2: (Deep learning training) The deep learning training process is implemented on the basis of the sample characteristics obtained according to Step 1 and the corresponding class labels, and meanwhile the deep encoding is adopted to further compress the characteristic dimensionality, specifically as mentioned in Algorithm 2.

Step 3: (Network test) The sparse encoding extraction function is adopted for similar test samples, and the deep learning model (Algorithm 2) constructed thereby is adopted to predict the class label of the test samples.

The calculation complexity of the algorithm is analyzed as follows:

According to the information theory, in the machine learning solution process based on inaccurate and incomplete information, algorithm complexity $P$ is directly proportional to $(1 / \varepsilon)^{n / r}$, namely:

$$
P \propto(1 / \varepsilon)^{n / r}
$$

Where ${ }^{\varepsilon}$ is the allowable value for machine learning; $n$ is the problem scale, and if the problem involves in the mapping function to be approximated, then $n$ can be regarded as the quantity of the independent variables of the mapping function to be approximated; $r$ is the smoothing parameter of the mapping function to be approximated, and smaller $r$ value indicates less smooth function relationship, namely more complicated mapping function relationship.

During the construction of the deep learning network model, due to the limited sample quantity and the determined noise influence on the samples, the sample information provided by the samples to the deep network is uncertain and incomplete. However, the complexity of the deep learning network training process can be attributed to the information complexity of the sample data, and we can obtain the following formula according to the analogical relationship:

$$
P^{\prime} \propto(1 / \varepsilon)^{n / R}
$$

Where ${ }^{\varepsilon}$ is the allowable value for machine learning and can be represented as the generalization training deviation during the deep network learning process; $n$ is the node quantity of the input layer; $\mathrm{R}$ is the relevance of the sample data or the mapping functions to be approximated, and can be represented as the smoothing parameter.

Additionally, larger complicated network indicates the stronger problem-solving ability thereof, and the problem-solving ability of the deep network can be measured according to capacity dimension index $d_{v c}$, wherein the lower estimation limit of capacity dimension index $d_{v c}$ is as follows:

$$
d_{v c} \geq 2\left[\frac{H}{2}\right] n
$$

Where $H$ is the node quantity of hidden layer and $n$ is the input dimensionality of the network. According to the above analysis, the key for constructing the deep network model is that network capacity index $d_{v c}$ should be matched with the complexity of the information data, namely: if the information data are more complicated, the capacity dimensionality index $d_{v c}$ for solving the problem should be larger. If only the single-node output is considered $(k=1)$, Formula (3) can be further approximated as follows:

$$
d_{v c} \geq(n+1) H
$$

The above formula can be further represented as follows: 


$$
C_{R}=\varepsilon[(n+1) H]^{R / n}
$$

Parameter $C_{R}$ of the formula is related to complexity parameter $\mathrm{R}$ of the problem to be solved, training operation error $\varepsilon$ and node quantity $\mathrm{H}$ of the hidden layer of the network.

\section{Experimental analysis}

\subsection{Experiment setting}

In consideration of a three-span bridge structure, the bridge is constructed through the tool cabinet of Matlab structural dynamics and is composed of 150 nodes and 192 elements, wherein the length and the width of the bridge surface are respectively assumed as $80 \mathrm{~m}$ and $8 \mathrm{~m}$, and the bridge is assumed to have two lanes. Additionally, the bridge is made of steels, with the Young modulus and the shearing modulus of elasticity respectively assumed as $210 \mathrm{GPa}$ and $80 \mathrm{GPA}$. The properties of main materials used in the experiment are as shown in Table 1. However, the bridge has been damaged or corroded, so the two parameters should be reduced according to the damage or corrosion degree. The system is stimulated by the uniform pressure acted on the whole bridge surface, and the bridge only has plane vibration. Moreover, left and right edges of the bridge surface and the bottoms of the three bridge piers are fixed.

Table 1. Steel Material Characteristic

\begin{tabular}{cccc}
\hline Symbol & Value & Unit & Physical Description \\
\hline $\mathrm{Nu}$ & 0.285 & & Poisson's ratio \\
$\mathrm{E}$ & 210000000000 & $\mathrm{~Pa}$ & Young's \\
$\mathrm{G}$ & 81712062256 & $\mathrm{~Pa}$ & Shear \\
Rho & 7800 & $\mathrm{Kg} / \mathrm{m} 3$ & Density \\
Alpha & 0 & $/ \mathrm{oC}$ & Thermal \\
Eta & 0 & Loss & factor \\
$\mathrm{T} 0$ & 20 & $\mathrm{oC}$ & Reference \\
\hline
\end{tabular}

In order to quantify bridge damage degree and bridge status derivative, four scenes are defined: one health scene and three damage scenes. The differences among these schemes lie in the Young modulus and the shearing modulus of elasticity at the preset positions: DL1, DL2 and DL3 (bridge surface between two bridge piers). For convenience, D1, D2 and D3 are used to represent the three damage scenes. Under the health condition, the Young modulus and the shearing modulus of elasticity are respectively as shown in Table 1. However, when the steel material is damaged along with the time, both the Young modulus and the shearing modulus of elasticity will be undoubtedly reduced. Specifically, the reductions of the Young modulus and the shearing modulus of elasticity at the three damage positions DL1, DL2 and DL3 are respectively as shown in Tables 2 and 3.

Table 2. Reduction of Young Modulus

\begin{tabular}{cccc}
\hline Damage Position & DL1 $(\%)$ & DL2(\%) & DL3(\%) \\
\hline D1 & 4 & 8.3 & 6 \\
D2 & 7 & 14 & 9 \\
D3 & 10 & 32 & 21 \\
\hline
\end{tabular}


Table 3. Reduction of Shearing Modulus of Elasticity

\begin{tabular}{cccc}
\hline Damage Position & DL1(\%) & DL2(\%) & DL3(\%) \\
\hline D1 & 2.4 & 7.4 & 6 \\
D2 & 6 & 14 & 9 \\
D3 & 11 & 31 & 21 \\
\hline
\end{tabular}

In practice, bridge conditions may be easily changed by the influence of the environment factors including wind, humidity, temperature and even slight disturbance, etc., so the accelerated velocity data collected by the sensors are changed. In order to more really carry out the experiment, all environment noises are assumed to follow Gaussian distribution with zero mean and standard deviation. Not all environment factors can be listed in the experiment and the dominate position of these factors cannot be judged, so the most reliable method is to assume that all factors follow Gaussian distribution. Based on the above assumption, for each sample data, the environment noise can be added as follows:

$$
\alpha_{i}(t)=\alpha_{i}(t)+N(0, \sigma)(t)
$$

Where $\alpha_{i}(t)$ is the accelerated velocity data measured by sensor $i$ at time $t$, and $N(0, \sigma)$ is Gaussian random variable with mean as 0 and variance as $\sigma ; \sigma=1$ and $\sigma=0.5$ are respectively considered in the experiment.

\subsection{Classification accuracy index}

BP neural network, Logistic regression [15], Softmax[16] and decision tree [17] are respectively selected as the comparison algorithms. All data are divided into two parts: $70 \%$ for training and 30\% for test. The simulation results are as shown in Figures 3 4, and the algorithm operation time comparison is as shown in Figure 5.

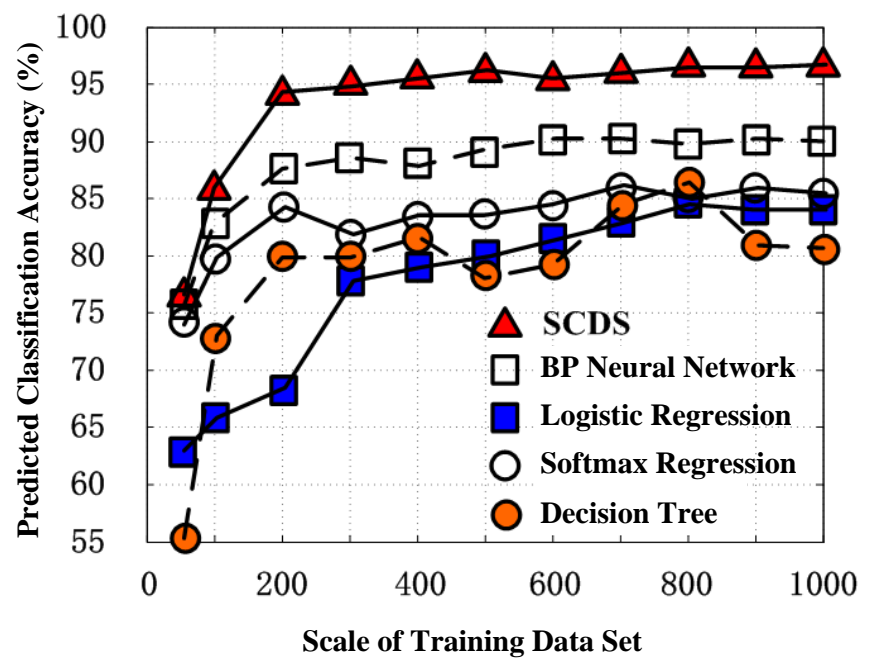

Figure 3. Classification accuracy $(\mathrm{N}(0,1))$ 


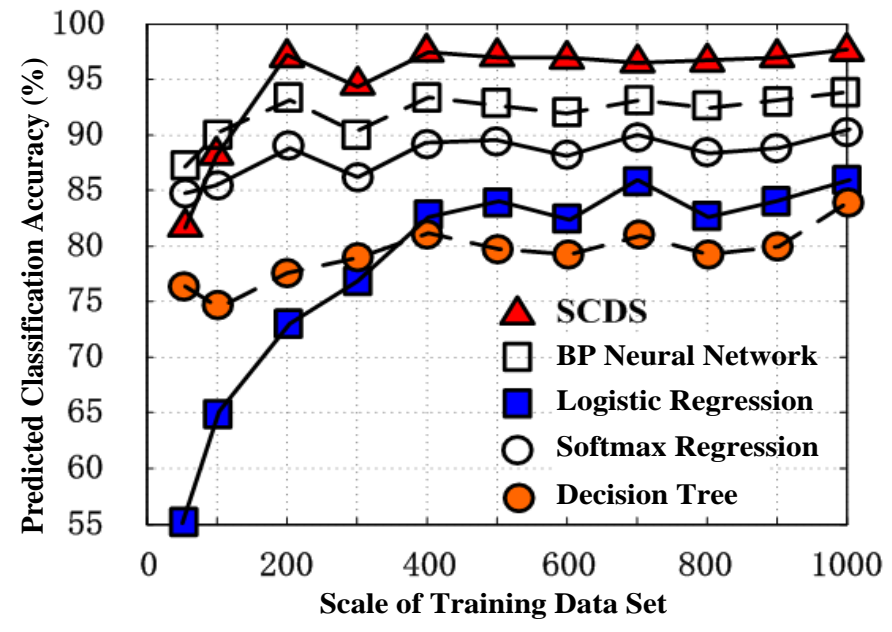

Figure 4. Classification accuracy $(\mathrm{N}(0,0.5))$

Figure 3 shows the change of the predicted classification accuracies of the comparison algorithms along with the scale change of the training data set under the noise of $N(0,1)$. Generally, the prediction accuracy of the proposed SCDC algorithm is higher than that of the comparison algorithms, and can be kept above 95\% when the training set has large scale. Among the comparison algorithms, BP neural network has relatively high prediction accuracy; Softmax regression, Logistic regression and decision tree algorithms have relatively poor algorithm effect.

Figure 4 shows the change of the predicted classification accuracies of the comparison algorithms along with the scale change of the training data set under the noise of $N(0,0.5)$. Generally, when the scale of the training set is less than 200, the prediction accuracy of the proposed SCDC algorithm is slightly lower than that of BP neural network and Softmax regression algorithms; under other conditions, the accuracy of SCDC algorithm is higher than that of the comparison algorithms and can be basically kept above 95\%. Among the comparison algorithms, the prediction accuracies of BP neural network and Softmax regression algorithms are relatively higher; Logistic regression and decision tree algorithms have relatively poor effect.

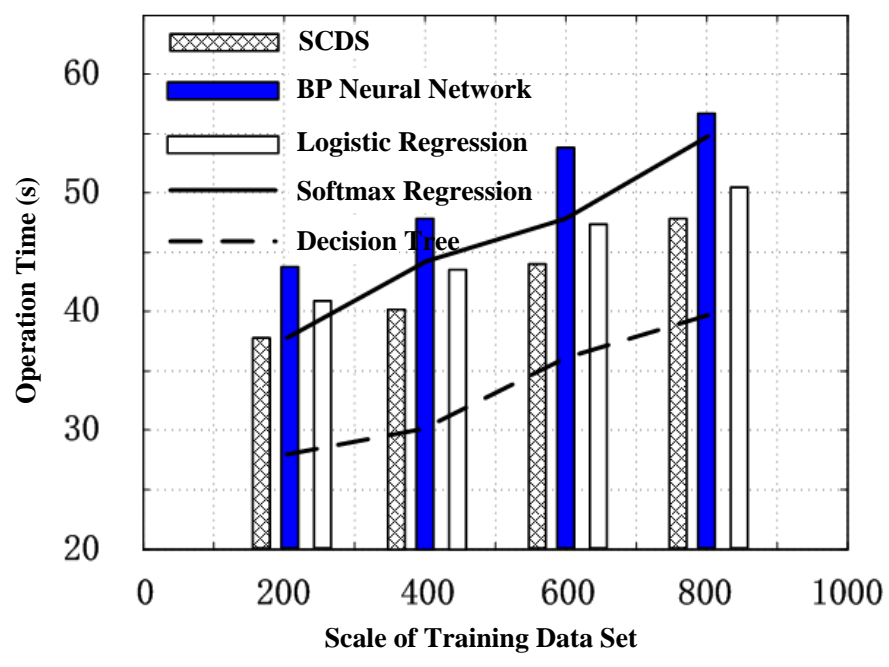

Figure 5. Operation time comparison 
Figure 5 shows the change of the operation time of the comparison algorithms along with the scale change of the training data set. According to the figure, the decision tree algorithm has the shortest calculation time due to the simple algorithm principle thereof, BP neural network algorithm has the longest operation time, and the proposed SCDC algorithm can take the second position, thus presenting certain calculation efficiency.

\section{Conclusion}

In allusion to the bridge structure health problem, wireless sensor network is configured and the sparse encoding deep learning algorithm model is established in this paper to realize the structural health monitoring. Meanwhile, the calculation performance of the deep learning algorithm is improved, and Hessian optimization is also improved on the basis of the linear conjugate gradient in order to replace uncertain Hessian matrix by positive semidefinite Gaussian - Newton curvature matrix for secondary objective combination, thus to improve the efficiency of the deep learning algorithm. The simulation result shows that the proposed algorithm is superior to the comparison algorithms in the aspect of the prediction accuracy and has certain calculation efficiency.

\section{Reference}

[1] Lv Z. Wearable smartphone: Wearable hybrid framework for hand and foot gesture interaction on smartphone[C]//Computer Vision Workshops (ICCVW), 2013 IEEE International Conference on. IEEE, (2013): 436-443.

[2] Lin Y, Yang J, Lv Z. A Self-Assessment Stereo Capture Model Applicable to the Internet of Things[J]. Sensors, (2015), 15(8): 20925-20944.

[3] Yang J, He S, Lin Y. Multimedia cloud transmission and storage system based on internet of things[J]. Multimedia Tools and Applications, (2015): 1-16.

[4] Lv Z, Yin T, Han Y. WebVR——web virtual reality engine based on P2P network[J]. Journal of Networks, (2011), 6(7): 990-998.

[5] Yang J, He S, Lin Y. Multimedia cloud transmission and storage system based on internet of things[J]. Multimedia Tools and Applications, (2015).

[6] Guo C, Liu X, Jin M. The research on optimization of auto supply chain network robust model under macroeconomic fluctuations[J]. Chaos, Solitons \& Fractals, (2015).

[7] Li X, Lv Z, Hu J. XEarth: A 3D GIS Platform for managing massive city information[C]//Computational Intelligence and Virtual Environments for Measurement Systems and Applications (CIVEMSA), 2015 IEEE International Conference on. IEEE, (2015): 1-6.

[8] Yang J, Chen B, Zhou J. A Low-Power and Portable Biomedical Device for Respiratory Monitoring with a Stable Power Source[J]. Sensors, 2015, 15(8): 19618-19632.

[9] Guanqun Bao, Liang Mi, Yishuang Geng, Kaveh Pahlavan, A computer vision based speed estimation technique for localiz ing the wireless capsule endoscope inside small intestine, 36th Annual International Conference of the IEEE Engineering in Medicine and Biology Society (EMBC), Aug. (2014).

[10] Xinchao Song, Yishuang Geng, Distributed community detection optimization algorithm for complex networks, Journal of Networks, 9(10), 2758-2765, Jan. (2014).

[11] Jiang D, Ying X, Han Y. Collaborative multi-hop routing in cognitive wireless networks[J]. Wireless Personal Communications, (2015): 1-23.

[12] Jinyu $\mathrm{Hu}$ and Zhiwei Gao. Modules identification in gene positive networks of hepatocellular carcinoma using Pearson agglomerative method and Pearson cohesion coupling modularity[J]. Journal of Applied Mathematics, 2012 (2012).

[13] Jiang D, Xu Z, Chen Z. Joint time-frequency sparse estimation of large-scale network traffic[J]. Computer Networks, 2011, 55(15): 3533-3547.Jinyu Hu, Zhiwei Gao and Weisen Pan. Multiangle Social Network Recommendation Algorithms and Similarity Network Evaluation[J]. Journal of Applied Mathematics, 2013 (2013). 


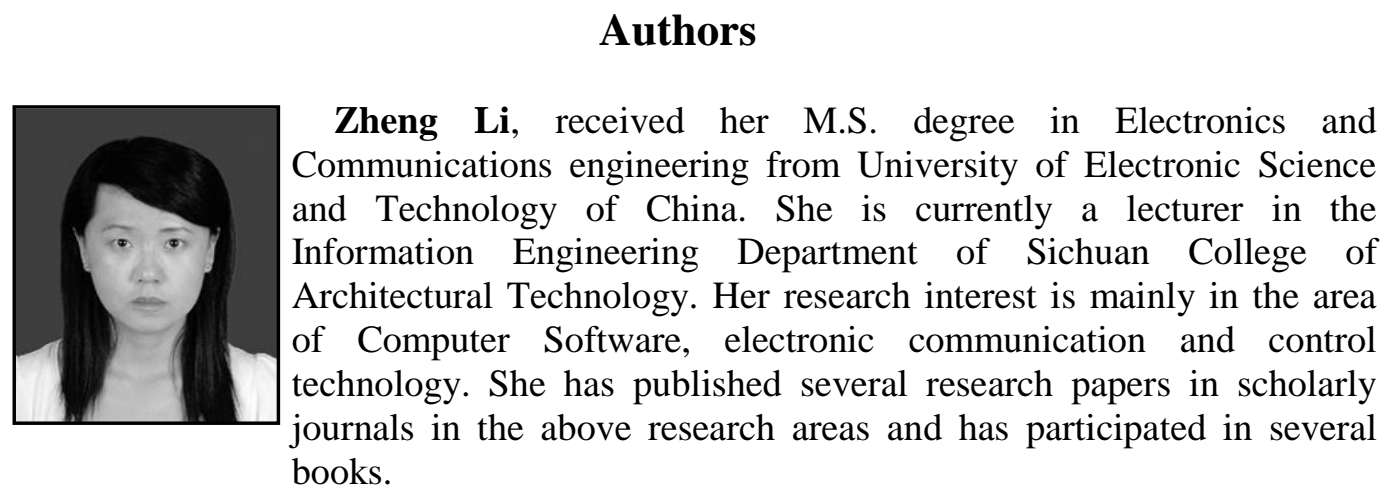

